\title{
Ionization and ablation phenomena in an ablative plasma accelerator
}

\author{
M. Keidar ${ }^{\text {a) }}$ and I. D. Boyd \\ Department of Aerospace Engineering, University of Michigan, Ann Arbor, Michigan 48109 \\ I. I. Beilis \\ Electrical Discharge and Plasma Laboratory, Tel Aviv University, Tel Aviv, P. O. Box 39040, \\ Tel Aviv 69978, Israel
}

(Received 22 March 2004; accepted 17 August 2004)

\begin{abstract}
Several interrelated phenomena near the surface ablated into a discharge plasma, such as ablation and ionization in accelerated plasma are studied. Two characteristic ablation modes are identified, namely, ablation mode with a velocity at the Knudsen layer edge smaller than the local sound speed and a velocity at the Knudsen layer edge close to the sound speed. The existence of these two ablation modes is determined by the current density in the acceleration region. The nonequilibrium ionization region in the presence of strong electromagnetic plasma acceleration is studied. In the subsonic regime, the ionization region thickness is proportional to the ionization rate and inversely proportional to the magnetic field. Conditions for ionization equilibrium in the accelerating plasma are determined. The specific example of a micropulsed plasma thruster is considered. It is concluded that both the equilibrium and nonequilibrium ionization regimes occur in this device. (C) 2004 American Institute of Physics. [DOI: 10.1063/1.1805726]
\end{abstract}

\section{INTRODUCTION}

In electrical discharges where plasma generation occurs at the surface such as a vacuum arc or an ablation controlled discharge (pulsed plasma thruster, capillary discharge, electroguns, circuit breakers, laser ablation devices, etc.) the plasma-wall interaction plays an extremely important role. ${ }^{1-6}$ The accurate description of the plasma-wall interaction is of high importance for theoretical investigations of such discharges, because it helps to formulate proper boundary conditions for the overall discharge model. There are several characteristic subregions near the surface in which different physical phenomena play a leading role, namely, spacecharge sheath, Knudsen layer, presheath, and a region where transition to ionization equilibrium occurs, or nonequilibrium ionization region (NEIR). These subregions constitute the entire transition region between the equilibrium plasma and the wall. The role of NEIR in the overall dynamics of the transition region is very important in order to calculate the energy and particle fluxes to the wall. ${ }^{7-9}$

There are some physical situations in which plasma is accelerated by the external forces in the region near the wall. An example of this is ablative pulsed plasma accelerator. ${ }^{10}$ In these accelerators, rapid heating of a thin dielectric surface layer leads to decomposition of the wall material. Ablative products are ionized in the vicinity of the dielectric and are accelerated by an electromagnetic force. Ionization of the ablative products and plasma acceleration take place in the same region and therefore both phenomena are interrelated. These phenomena are interesting from the standpoint of smooth transition between the two ablation modes with small (subsonic) and sonic plasma velocity at the edge of the Knudsen layer that strongly affect ablation. ${ }^{11}$ The conditions at the Knudsen layer edge are very important for calculation of the flux of returned particles to the surface. For instance it

${ }^{a)}$ Electronic mail: keidar@engin.umich.edu was shown that the backflux is responsible for surface carbonization in the case of Teflon ${ }^{\mathrm{TM}}$ ablation. ${ }^{12}$ The effect consists in the flux of returned particles to the surface that is determined by the bulk plasma density and temperature. In principle two limits are possible. When plasma density is very high the flux of returned particles is strong. As a result the velocity at the edge of the Knudsen layer and the corresponding ablation rate are small. The opposite limit corresponds to the case when plasma density is small. The actual limit for the high and small plasma density is determined by specific conditions of the experiment, but is close to the equilibrium vapor density. The plasma density and temperature depend on the flow conditions in the plasma bulk. One can expect that there should be smooth continuous transition between these two regimes. In small plasma devices, the spatial extension of the ionization layer may become comparable to the size of the device. An example of such a device is a micropulsed plasma thruster. ${ }^{13}$ The conditions of ionization equilibrium in the presence of strong plasma acceleration were not addressed previously and therefore require special attention. In this paper, the phenomena associated with contact between the surface and plasma under conditions of strong plasma acceleration are considered taking into account neutral generation (ablation) and neutral ionization.

The rest of the paper is organized as follows. In Sec. II, the problem of surface ablation into a discharge plasma is formulated in the general case. The model of the NEIR under the condition of strong plasma acceleration is developed in Sec. III. Application of the results to the specific physical situation of a micropulsed plasma thruster is considered in Sec. IV.

\section{ABLATION PHENOMENA AND THE KNUDSEN LAYER}

In this section, the problem of evaporation under the condition of strong plasma acceleration near the ablated sur- 


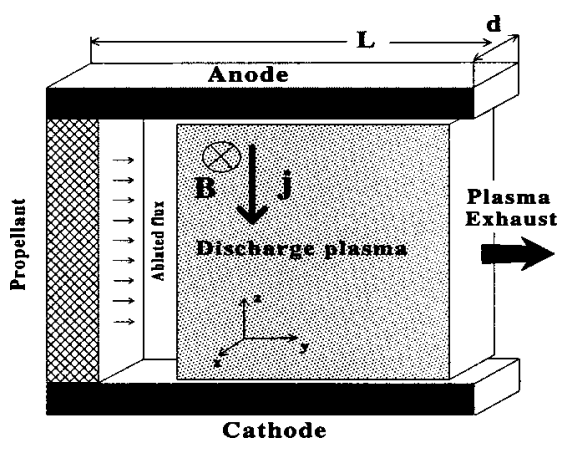

FIG. 1. Schematic of the electromagnetic pulsed plasma thruster.

face is studied. An example of a pulsed plasma thruster ${ }^{10-13}$ is considered (see Fig. 1). In the pulsed plasma thruster, a discharge is struck between two electrodes across the dielectric (propellant). Propellant is heated during the discharge by the plasma and ablates. The ablated vapor is ionized in the vicinity of the propellant and is accelerated by the electromagnetic force.

Similarly to the previous work, ${ }^{11}$ we consider the multilayer structure of the near surface region (see Fig. 2). One can distinguish two different characteristic regions between the surface and the plasma bulk:

(1) A kinetic nonequilibrium layer adjacent to the surface with a thickness of a few mean free paths (the Knudsen layer); boundary 1 corresponds to the Knudsen layer edge.

(2) A collision-dominated (hydrodynamic) region; boundary 2 constitutes the edge of the hydrodynamic region.

The definitions for the edge of the Knudsen layer and the hydrodynamic region are considered below.

We start from a formulation of the equation set for the hydrodynamic region. For our primary purpose of considering the ablation effects, we simplify the present analyses and assume that ionization equilibrium is reached near the edge of the hydrodynamic region (boundary 2). Detailed analysis of the ionization phenomena is presented in the following section. Here we assume that the thickness of the NEIR is smaller than the thickness of the hydrodynamic region. The specific conditions under which this assumption is justified are considered in the following section. In this case the electron density at the boundary 2 will be calculated using Saha equilibrium. In the typical range of parameters (plasma density is about $10^{23}-10^{24} \mathrm{~m}^{-3}$ and electron temperature is about $2-4 \mathrm{eV}$ ) almost fully ionized plasma is expected according to Saha equilibrium. The plasma parameters $(n, T)$ in
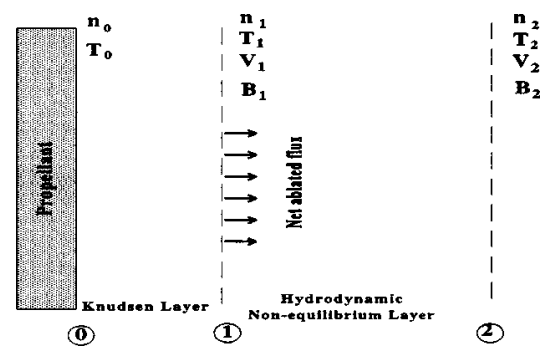

FIG. 2. Schematic of the near surface layers. the hydrodynamic region depend on the conditions at the boundary 1, which is the Knudsen layer edge. To find the parameters at the edge of the Knudsen layer as a function of velocity at the Knudsen layer edge $V_{1}$ we use the mass, momentum, and energy conservation equations in accordance with relations (listed in the Appendix) between parameters at the edge of the Knudsen layer and those at the surface presented in Refs. 14-16 and compared with Monte Carlo simulation in Ref. 17.

First, the simple model for the heavy particles (including ions and atoms) flow is considered. Starting from the boundary 1 , in the hydrodynamic region we apply the conservation laws for mass, momentum, and energy. We will consider a simplified, single fluid magnetohydrodynamics (MHD) approximation taking into account the self-magnetic field. The magnetic field has a primary component in $x$ direction as shown in Fig. 1 and decreases between boundaries 1 and 2 due to magnetic diffusion. The mass conservation equation for the heavy particles,

$$
\frac{d(n V)}{d y}=0,
$$

where $n$ is the plasma density and $V$ is the velocity. The momentum conservation equation for the plasma,

$$
M(n V) \frac{d V}{d y}=-\frac{d(n k T)}{d y}+j B,
$$

where $M$ is the atomic mass, $T$ is the plasma temperature, $j$ is the current density, and $B$ is the magnetic field. The relation between current density and magnetic field can be determined from Faraday's law:

$$
j=-\frac{1}{\mu} \frac{d B}{d y} .
$$

Substituting Eqs. (1) and (3) into Eq. (2) and integrating Eq. (2) one can obtain the following relationship between parameters at the boundaries 1 and 2 :

$$
n_{1} k T_{1}+M n_{1} V_{1}^{2}+\frac{1}{2} \frac{B_{1}^{2}}{\mu}=n_{2} k T_{2}+M n_{2} V_{2}^{2}+\frac{1}{2} \frac{B_{2}^{2}}{\mu} .
$$

The plasma density and velocity distribution in the hydrodynamic region as well as the values of $B_{1}$ and $B_{2}$ depend on the specific geometry of the accelerator. Let us consider a planar geometry as an example (see Fig. 1). Further analyses will be based on additional assumptions regarding the plasma acceleration in the electromagnetic thruster. Using the MACH2 computer code, it was demonstrated that the magnetosonic condition exists at the thruster exit plane. ${ }^{18}$ The magnetosonic condition is associated with the Alfvén critical speed $V_{c} \cdot{ }^{19}$ This velocity is proportional to the ionization potential, $V_{c}=\left(2 e U_{i} / M\right)^{0.5}$, where $U_{i}$ is the ionization potential. Originally, Alfvén put this idea forward ${ }^{20}$ in his theory on the origin of the solar system. The idea states that if the relative velocity between plasma and neutral gas in a magnetic field reaches or exceeds a certain critical value, the gas is efficiently ionized. This velocity is determined by the condition that the ion kinetic energy is equal to the ionization energy of the gas. Sometimes this relation is called the 
Alfvén hypothesis and is used in the model of plasma acceleration in a pulsed plasma thruster (PPT) (Ref. 21). The Alfvén hypothesis was experimentally verified and shown to be correct. ${ }^{22}$ Recent simplified model of the plasma acceleration showed that the Alfvén sound speed is proportional to the Alfvén critical velocity and is about $50 \%$ higher. ${ }^{19}$ Also recent simulations using MACH2 shows (Ref. 18) that the flow speed near the exit of the thruster equals the local value of the Alfvén wave speed. It was shown that in the framework of the MHD approach, there exists the so-called magnetosonic condition i.e., plasma accelerates up to the velocity equal to the Alfvén sound speed. Based on the above we will make here an assumption about the existence of a magnetosonic point where the flow velocity equals Alfvén's wave speed. The assumption about the magnetosonic point allows us to reduce the number of unknowns in the problem. We take the boundary 2 at the distance where the plasma velocity $V_{2}$ approaches the Alfvén velocity. In other words, this assumption is equivalent to the condition that most of the plasma acceleration takes place in the region between boundaries 1 and 2 and that the kinetic energy density approaches the magnetic energy density at the boundary 2 ,

$$
V_{2}=\frac{B_{2}}{\left(\mu n_{2} M\right)^{0.5}},
$$

where $n_{2}$ is the electron density at the boundary 2 . To close the system of equations for the hydrodynamic layer, and taking into account that the magnetic field decreases along the channel, we can estimate the magnetic field at the edge of the Knudsen layer (boundary 1) as

$$
B_{1}=\mu I / d,
$$

where $d$ is the characteristic length of the electrode width (Fig. 1) and $I$ is the current. Combining Eqs. (4)-(6) one can obtain the following expression for the velocity at the outer boundary of the kinetic (Knudsen) layer:

$$
\frac{M V_{1}^{2}}{2 k T_{1}}=\frac{\frac{n_{1}}{2}-\frac{T_{2} n_{2}}{2 T_{1}}+\frac{1}{4} \frac{\mu(I / d)^{2}}{k T_{1}}}{\frac{3}{2} \frac{n_{1}^{2}}{n_{2}}-n_{1}} .
$$

One can see that the velocity at the edge of the Knudsen layer depends upon density at the exit plane (boundary 2), electrode geometry $(d)$, and current. In the case of $I=0$ (i.e., electrothermal thruster or ablative capillary discharge) this equation reduces to the one obtained previously [Eq. (4) in Ref. 11].

In order to study the general trends of the dependence of the velocity at the Knudsen layer edge $V_{1}$ on the current density without details of the flow field along the channel we consider the plasma density at the magnetosonic plane (boundary 2, Fig. 2) as a parameter. The calculated velocity $V_{1}$ is shown in Fig. 3 as a function of current density. Parameters $\left(T_{1}\right.$ and $\left.n_{1}\right)$ at the Knudsen layer edge (boundary 1) are calculated using Knudsen layer relations (Appendix). Heavy particle density distribution along the acceleration channel depends upon the specific geometry of the channel (for instance the length of the channel and interelectrode dis-

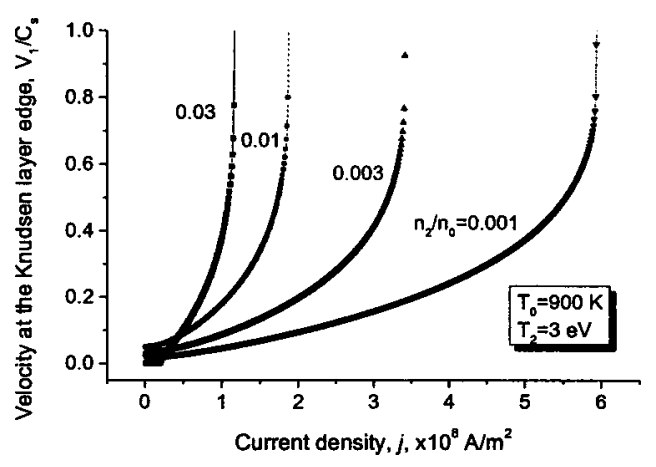

FIG. 3. Velocity at the edge of the Knudsen layer as a function of current density with density at the edge of hydrodynamic layer as a parameter. $L$ $=d=1 \mathrm{~cm}$.

tance as shown in Fig. 1). One can see that the solution with a small current density corresponds to small velocity in comparison to the sound speed. The calculation shows that the value $V_{1}$ is close to that obtained in the case of an electrothermal plasma accelerator ${ }^{23}$ and corresponds to ablation into the dense background plasma ${ }^{1,11,15}$ when $I=0$. The velocity $V_{1}$ increases with current density and approaches the sound speed limit at some high current density dependent on the plasma density at the boundary 2. According to the model, the electromagnetic force in the region between boundaries 1 and 2 leads to significant plasma acceleration that in turn affects the plasma parameters $\left(n_{1}\right.$ and $\left.T_{1}\right)$ at boundary 1 . At some value of current density, the solution approaches the limit of the local sound speed. Note that no solution exists beyond the case when velocity $V_{1}$ equals the sound speed, since no physical mechanism for supersonic acceleration in the Knudsen layer exists. Therefore, density $n_{2}$ when $V_{1}$ $=C_{\mathrm{S}}$ can be considered as a limiting possible density under the considered conditions. This means that a combination of the current density and the geometry (that determine the flow field, i.e., $n_{2}$ ) uniquely determine the solution. This rather general model (in which three parameters are considered as known) can be coupled with a model of the plasma flow and details of the energy conservation in the particular system. In this case the number of free parameters will be reduced and a self-consistent solution can be obtained. An example of such a model in the case of an electrothermal device was presented earlier. ${ }^{23}$

Plasma acceleration in the hydrodynamic layer causes transition from the ablation mode with small velocity at the Knudsen layer edge and significant backflux to the ablation mode with sonic velocity at the Knudsen layer edge, which is the situation for ablation into vacuum. When the plasma velocity is sufficiently small ${ }^{1,15,23}$ it can be assumed that equilibrium ionization (according to Saha equation) takes place. However, when strong plasma acceleration takes place in the hydrodynamic region, this may affect equilibrium conditions. In the following section we will consider ionization processes under the effects of plasma acceleration.

\section{IONIZATION IN THE PRESENCE OF PLASMA ACCELERATION IN THE HYDRODYNAMIC REGION}

We start our consideration from the Knudsen layer edge. The reason for this is that in the typical conditions the mean 
free path is much larger than the Debye length and therefore the sheath thickness is much smaller than that of the Knudsen layer length. This simplifies the problem significantly since the boundary conditions are known at the Knudsen layer edge (see the Appendix). By definition the ionization region is collision dominated and therefore hydrodynamic approximation can be used. Considering that the thickness of the ionization region is much smaller than the transversal dimensions, the system of governing equations in a onedimensional form can be written as

$$
\begin{aligned}
& \frac{d\left(n_{i} V_{i}\right)}{d y}=\alpha_{i} n_{i} n_{a}-\alpha_{r} n_{i}^{2}, \\
& n_{i} k\left(T_{h}+T_{e}\right)+n_{a} k T_{h}=P, \\
& M n_{i} V_{i} \frac{d V_{i}}{d y}=-\frac{d\left(P_{i}+P_{e}\right)}{d y}-\frac{1}{2 \mu} \frac{d\left(B^{2}\right)}{d y}-M \alpha_{i} n_{i} n_{a} V_{i}, \\
& j=\sigma(E+V B)=-\frac{1}{\mu} \frac{d B}{d y},
\end{aligned}
$$

where $\alpha_{i}$ is the ionization rate, $\alpha_{r}$ is the recombination rate, $n_{i}, n_{a}$ are ion and neutral density, $T_{e}, T_{h}$ are electron and heavy particles (ions, atoms) temperature. $P_{i}, P_{e}, P$ are ion, electron, and total pressure, respectively. We will use the equation of state for an ideal gas (i.e., $P=n k T$ ). Due to the high electron conductivity, electron temperature variation across the ionization layer is expected to be small and is neglected. We will assume that the ionization rate determines the length of the ionization layer and therefore for simplicity recombination is neglected. As a first approach we will consider plasma flow with uniform current distribution that approximately corresponds to the slow moving current stage of a pulsed plasma thruster. ${ }^{10,24}$ This means that we assume that $j=\sigma(E+V B)=$ const.

We use the following dimensionless variables: $\xi$ $=y \alpha_{i} n_{i o} / C_{i a}, b=B / B_{1}, n=n_{i} / n_{i o}, v=V_{i} / C_{s}$, where $C_{i a}$ is the heavy particle thermal velocity, $B_{1}$, is the magnetic field at the edge of the Knudsen layer, $n_{i o}$, is the plasma density in the hydrodynamic region at the edge of the NEIR. In the absence of acceleration, the NEIR edge corresponds to full ionization, i.e., $n=1$. This density is determined by the conditions in the plasma bulk. By NEIR definition, this density can be determined from equilibrium analyses. Taking the above into account, the system reduces to the following:

$$
\begin{aligned}
& \frac{d b}{d \xi}=-\alpha, \\
& \left(v^{2}-1\right) \frac{d v}{d \xi}=-\left(v^{2}+1\right) \varepsilon^{0.5}(1-n)-\frac{v}{2 n} \beta \frac{d(b)^{2}}{d \xi}, \\
& v \frac{d n}{d \xi}=-n \frac{d v}{d \xi}+n \varepsilon^{0.5}(1-n),
\end{aligned}
$$

where $\alpha=C_{i a} /\left(d \alpha_{i} n_{i o}\right), \beta=\left(V_{\alpha} / C_{s}\right)^{2}, \varepsilon=\left(T_{i}+T_{e}\right) / T_{i}$, and $V_{a}$ $=\left(B_{1} / \mu m n_{i a}\right)^{0.5}$, and $d$ is the electrode width. For this system of equations, the following boundary conditions can be used: $n=0$ and $b=1$. Another additional boundary condition is the velocity at the edge of the hydrodynamic region. It was shown in the preceding section that the velocity at the edge of the Knudsen layer varies in the range from very small $v$ $\ll 1$ up to the local sonic speed. Note that the velocity at the Knudsen layer edge (shown in Fig. 3) is normalized by the local sound speed determined by the heavy particle temperature at the Knudsen layer edge. This temperature is close to the surface temperature and much smaller than that of the electrons in the ionization layer. Therefore, a physically reasonable condition for the velocity is $v=0$ when $\xi=0$.

\section{A. Limit of small plasma acceleration}

First let us consider an asymptotic solution for the case of small plasma acceleration $\left(v^{2} \ll 1\right)$. In this case Eq. (13) reduces to

$$
\frac{d v}{d \xi}=\varepsilon^{0.5}(1-n)+\frac{v}{2 n} \beta \frac{d(b)^{2}}{d \xi} .
$$

By substitution of this equation and Eq. (12) into Eq. (14) we have

$$
\frac{d n}{d \xi}=\alpha \beta(1-\alpha \xi)
$$

The last equation has a solution in the form

$$
n=\alpha \beta \xi-0.5 \alpha^{2} \beta \xi^{2} \text {. }
$$

One can see that the problem is determined by two parameters $\alpha$ and $\beta$. Therefore, the region thickness where $n=1$ can be determined as a solution of this equation,

$$
\xi^{*}=\frac{1}{\alpha}\left(1-\sqrt{1-\frac{2}{\beta}}\right),
$$

in the most common case of $\beta \gg 1$ the last expression can be approximated as

$$
\xi^{*} \approx 1 /(\beta \alpha),
$$

where $\xi^{*}$ is the thickness of the ionization layer.

\section{B. Regular sonic transition}

In this section we describe the asymptotic behavior of the solution in the case of strong acceleration, i.e., $v \rightarrow 1$. Let us first calculate the plasma density near the sonic transition place $(v=1)$. The physically meaningful solution with smooth transition requires that the right-hand side of Eq. (13) be zero at the sonic plane. In general, using L'Hopital 's rule one can find the velocity gradient near the sonic plane, which is finite. This procedure was used by a number of authors (see Refs. 25 and 26). The numerator on the right-hand side of Eq. (13) has the following form in this case:

$$
0=-\left(v^{2}+1\right) \varepsilon^{0.5}(1-n)-\frac{v}{2 n} \beta \frac{d(b)^{2}}{d \xi} \text {. }
$$

Using the above assumption about constant current density one can find from Eq. (12) that $(1 / 2)\left[d(b)^{2} / d \xi\right]=-\alpha(1$ $-\alpha \xi)$. Taking this into account the equation for the density reads 

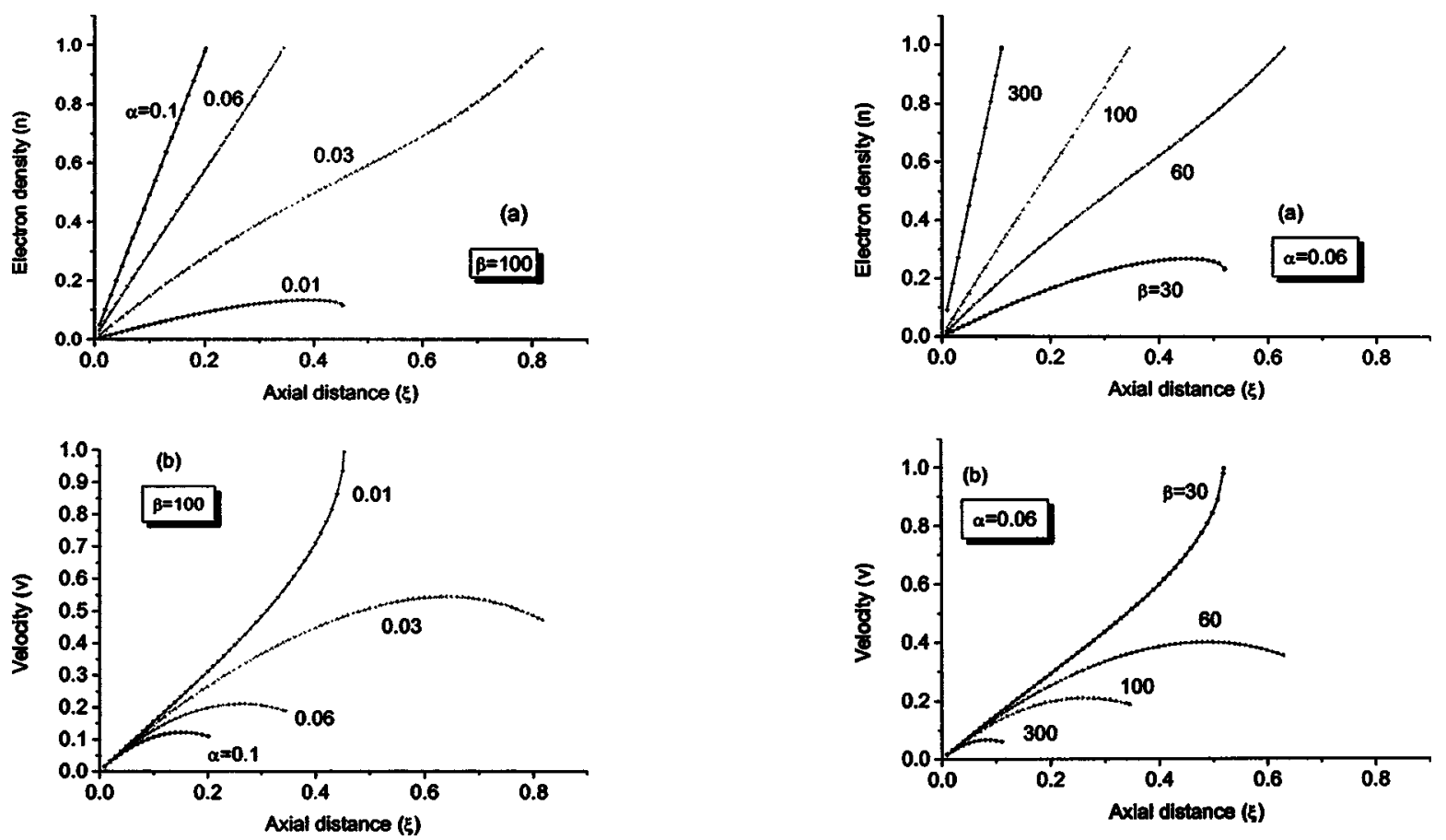

FIG. 4. Plasma density and velocity distribution in the ionization layer with $\alpha$ as a parameter. $\varepsilon=10$.

$$
n^{2}-n+\frac{\alpha \beta}{2 \varepsilon^{0.5}}(1-\alpha \xi) .
$$

Considering that the ionization layer thickness does not change significantly near the sonic transition region (as shown below) we can approximate the thickness as $\sim 1 / \alpha \beta$ [according to Eq. (19)]. Taking all the above into account, finally the physically meaningful solution that allows smooth transition has the following expression for density in the vicinity of the sonic point:

$$
n=0.5\left[1-\sqrt{1-\frac{2 \alpha \beta}{\varepsilon^{0.5}}\left(1-\frac{1}{\beta}\right)}\right] .
$$

This is an expression for density behavior at the sonic plane in the case of regular sonic transition. One can see that a small degree of ionization due to significant convection (plasma acceleration) $(n<1)$ can be expected if the function $2 \alpha \beta(1-1 / \beta) / \varepsilon^{0.5}<1$ as will be shown in the following section.

\section{Numerical examples}

In this section we describe some numerical examples of the solution of the ionization layer problem [Eq. (12)-(14)] which depends on three parameters $\alpha, \beta$, and $\varepsilon$. These calculations are shown in Figs. 4 and 5. Plasma density and velocity in the ionization layer with $\alpha$ as a parameter are shown in Fig. 4. By definition, the parameter $\alpha$ is inversely proportional to the ionization rate. One can see that when $\alpha$ is large, the ionization layer thickness (determined by condition $n=1$ ) becomes smaller. This happens because in the subsonic flow considered here, collisions (ionization in our case) lead to acceleration (which is usual for any one-dimensional gas flow). When $\alpha$ is small enough $(\alpha<0.03)$ the ionization

FIG. 5. Plasma density and velocity distribution in the ionization layer with $\beta$ as a parameter. $\varepsilon=10$.

equilibrium condition cannot be met. This is shown in the bottom of Fig. 4, where plasma velocity in the ionization layer is plotted. It can be seen that the plasma accelerates up to the sonic velocity when $\alpha$ is small before ionization equilibrium can be reached.

Similarly, a dependence on the parameter $\beta$ is found. Parameter $\beta$ represents an effect of the electromagnetic acceleration. When $\beta$ is large, plasma acceleration is small in the subsonic region and as a result ionization equilibrium can be reached.

One can conclude that there is a range of parameters when equilibrium ionization can be achieved, i.e., when $\beta>60$ and $\alpha<0.03$. These conditions are necessary conditions for the existence of the ionization equilibrium near the ablated surface under strong acceleration effects. An important conclusion that can be derived is that in order to have significant ionization, the ionization and accelerations regions must be separated. It should also be noted that the above numerical results are obtained considering only the subsonic region and therefore a singularity is found. In reality, this singularity will disappear if the condition for regular sonic transition is taken into account.

In this case, the electron density is calculated according to Eq. (22). In the following section, we apply this model for the specific case of a micropulsed plasma thruster ( $\mu$-PPT), where the driving parameters are determined for a specific situation and also current density is calculated instead of using a simplified approach.

\section{APPLICATION TO THE CARBON-FLUORINE PLASMA IN A MICROPULSED PLASMA THRUSTER}

In this section we apply the above results to a particular plasma device - a micropulsed plasma thruster ( $\mu$-PPT). This is a simplified version of a pulsed plasma thruster that 


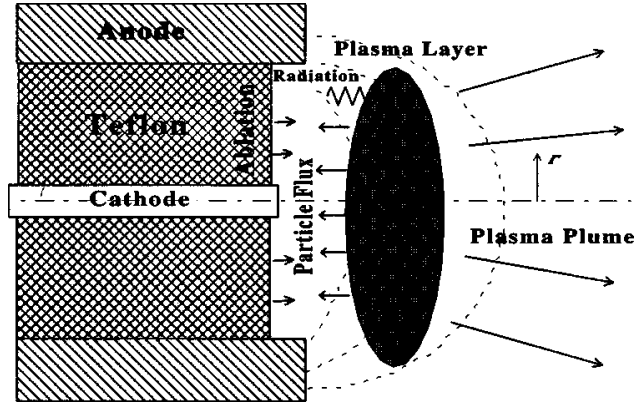

FIG. 6. Schematic of the plasma-surface interaction in a micropulsed plasma thruster.

was recently developed at the Air Force Research Laboratory (AFRL) for delivery of very small impulse bit. ${ }^{13}$ In order to make analyses of this particular device, we estimate the parameter $\beta$ and we calculate the current density distribution in the vicinity of the ablated surface instead of using an assumption about constant current density. The main objective of considering this particular example is to show under which conditions the effect of nonequilibrium ionization due to plasma acceleration is important. The characteristic ionization and recombination times are less than typical time for discharge parameter change in PPT (Refs. 2 and 23) and therefore steady state ionization model developed in Sec. III is employed.

The schematic of the plasma layer near the ablated surface is shown in Fig. 6. The plasma is created near the surface and the plasma layer is separated from the surface by the ionization layer described above. In order to calculate plasma properties we develop a model that includes Joule heating, heat transfer from the plasma to the wall, and dielectric (Teflon) ablation. Mechanisms of the energy transfer from the plasma include heat transfer by particle convection and radiation. It is assumed that within the plasma layer all parameters vary in the radial direction as shown in Fig. 6. The energy balance equation can be written in the following form:

$$
\frac{3}{2} n_{e} d T_{e} / d t=Q_{J}-Q_{r}-Q_{F},
$$

where $Q_{j}$ is the Joule heat, $Q_{r}$ is the radiation heat, and $Q_{F}$ is the heat associated with particle flux.

According to Ref. 27, radiation in the continuum from a $\mathrm{C}+2 \mathrm{~F}$ plasma in the considered parameter range provides the main contribution. The radiation energy flux $Q_{r}$ includes the radiation for a continuum spectrum based on a theoretical model. ${ }^{28,29}$ In the expression for $Q_{r}=A Z_{i}^{2} n_{e}^{2} T^{1 / 2}\left(1+\chi_{g}\right)$, the coefficient $\mathrm{A}$ is a constant $\left(1.6 \times 10^{-38}\right.$ in SI units) and $\chi_{g}$ $=E_{g} / T_{p}$ with $E_{g}$ as the energy of the low excited state. The particle convection flux $Q_{F}$ includes energy associated with electron and ion fluxes to the dielectric wall that leads to plasma cooling. It was shown previously that the energy is carried off mainly by the particle convection. ${ }^{2,23}$

The full system of equation is described in detail in Refs. 2 and 23. The general procedure of calculation of the plasma properties consists of the following. Electron temperature is calculated from the energy balance. For known plasma density and electron temperature, the heat flux to the surface is calculated. In turn, the surface temperature is calculated from the heat transfer equation with boundary conditions that take into account vaporization heat and conductivity. The solution of the heat transfer equation is considered for two limiting cases of small and large ablation rate similar to that described in Ref. 2. The density at the Teflon surface is calculated using the equilibrium pressure equation for Teflon and the density at the Knudsen layer edge is determined in the framework of the kinetic model (see the Appendix). For known pressure and electron temperature one can calculate the chemical composition of the $\mathrm{C}+\mathrm{F}$ plasma assuming local thermodynamic equilibrium ${ }^{2,23}$ (LTE) or using the nonequilibrium ionization approach developed in the previous sections. The results of calculation according to these two models will be compared. Using this approach the electron temperature and Teflon surface temperature can be calculated.

In order to calculate current density in the near field (as shown in Fig. 6) we assume that the magnetic field has only an azimuthal component and also neglect the displacement current. The main plasma density gradient developed in the axial direction and therefore gradient mechanism ${ }^{30}$ should not affect magnetic transport in this system. The combination of the Maxwell equations and electron momentum conservation gives the following equation for the magnetic field in the case of isothermal flow:

$$
\begin{aligned}
\partial \boldsymbol{B} / \partial \mathrm{t}= & 1 /(\sigma \mu) \boldsymbol{\nabla}^{2} \boldsymbol{B}-\boldsymbol{\nabla} \times\left[\boldsymbol{j} \times \boldsymbol{B} /\left(e n_{e}\right)\right] \\
& +\boldsymbol{\nabla} \times(\boldsymbol{V} \times \boldsymbol{B}) .
\end{aligned}
$$

A scaling analysis shows that the various terms on the righthand side of Eq. (24) may have importance in different regions of the plasma plume and therefore a general and endto-end plasma plume analysis requires keeping all terms in the equation. In the case of the near plume of the $\mu$-PPT with a characteristic scale length of about $1 \mathrm{~cm}$, the magnetic Reynolds number $\operatorname{Re}_{m} \ll 1$ and therefore the last term can be neglected.

In addition our estimations show that the Hall parameter is small $((\omega t \ll 1)$ if the plasma density near the Teflon surface $n_{e}>10^{23} \mathrm{~m}^{-3}$. This case is realized in the $\mu$-PPT so the Hall effect is expected to be small for this particular case. Therefore all results presented below are calculated from simple magnetic diffusion equation without considering the Hall effect, i.e., the second term in the right-hand side of Eq. (24) can also be neglected.

The following boundary conditions are employed. We assume that the current is uniform on both electrodes that allows us to estimate the current density on the cathode $j_{c}$ and on the anode $j_{n}$. The magnetic field is assumed to vary as $1 / r$ on the upstream boundary between the electrodes. At the lateral boundary we assume that the normal current $j_{n}=0$. The downstream boundary is considered to be far enough away so that $B=0$ can be assumed and finally along the centerline the magnetic field is zero. More details about this model can be found elsewhere. ${ }^{12,31}$ From the magnetic transport equation [Eq. (24)], the magnetic field and current density distributions can be calculated. In order to calculate the 


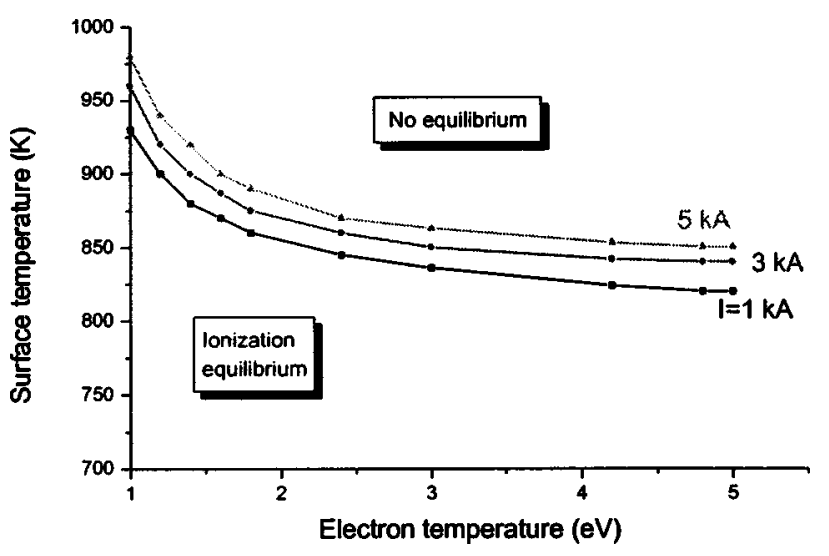

FIG. 7. Separation between two regions with equilibrium and nonequilibrium ionization conditions.

ionization layer properties near the Teflon surface, we will use the magnetic field distribution in the plume instead of assuming $j=$ const as was done in the above.

\section{A. Results}

In order to calculate the ionization rate for the $\mathrm{C}-\mathrm{F}$ plasma, the electron impact ionization cross sections (available from a database ${ }^{32}$ ) are used. In these calculations a specific example is considered. ${ }^{12,13}$ The micropulsed plasma thruster geometry and scale is shown in Fig. 6. It has a central electrode (cathode) with radius of about $0.4 \mathrm{~mm}$ and an outer electrode (anode) with inner radius of about $1.5 \mathrm{~mm}$. The ablative propellant is Teflon ${ }^{\mathrm{TM}}$. In these simulations, the experimental current wave form is used and it can be described in a first approximation as an underdamped LCR circuit current,

$$
I(t)=I_{p} \sin (\Omega t) \exp (-\gamma t),
$$

where $I_{p}=\sqrt{2 E / L}, \Omega=\sqrt{1 / L C}, \gamma=R / 2 L, L$ is the effective inductance in the circuit, $C$ is the capacitance, $R$ is the total circuit resistance, and $E$ is the pulse energy. Results presented below correspond to $E=2.25 \mathrm{~J}$ and $C=0.5 \mu \mathrm{F}$. The best fit with the experimental wave form ${ }^{12,13}$ (frequency) corresponds to $\Omega=4.7 \times 10^{7} \mathrm{rad} / \mathrm{s}$ and circuit inductance $L$ $=90 \mathrm{nH}$.

First, we calculate the ionization layer properties [according to Eqs. (12)-(21)] to understand if ionization equilibrium is achieved. In the Teflon surface temperatureelectron temperature $\left(T_{s}-T_{e}\right)$ plane, these dependencies are shown in Fig. 7. It can be seen that in general both regimes with ionization equilibrium and without it can be achieved in the considered range of parameters. One can see that the separation curve depends upon the total current in the discharge. For comparison we calculate the $T_{s}-T_{e}$ dependence from plasma layer model (see previous sections). These data are shown in Fig. 8. It follows from comparison of Figs. 7 and 8 that during the discharge there are regimes where equilibrium cannot be achieved. Therefore, the plasma composition in the plasma layer cannot always be calculated based on LTE assumption but rather the more general model [Eqs. (12)-(21)] should be employed.

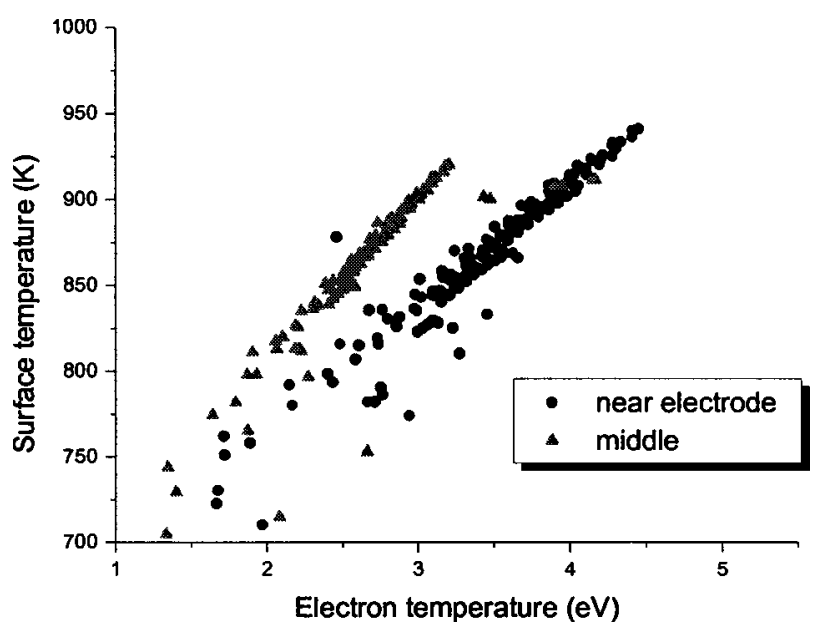

FIG. 8. Calculated dependence of the surface temperature and electron temperature. Sampling was taken at two locations, midway between the electrodes and near the outer electrode.

\section{SUMMARY}

In this paper we studied several interrelated phenomena near the surface ablated into a discharge plasma, such as ablation, formation of the ionization layer, and plasma generation. We found that there is a smooth transition between two ablation modes in the pulsed plasma accelerator, namely, one mode with a small velocity at the Knudsen layer edge and one with large (sound) velocity at the Knudsen layer edge. In the first case, the fraction of backflux is very large, while in the second case it is about $18 \%$ (16\% according to Monte Carlo simulations) of the primary flux. It was concluded that the transition between the two modes occurs as current density increases. The current density, which corresponds to this transition, depends on accelerator geometry and details of the flow in the acceleration channel. A model of the nonequilibrium ionization layer was developed. In two limiting cases, namely, small acceleration and smooth sonic transition, the analytical solution was obtained. It was found that plasma acceleration significantly alters the ionization region. The model of the nonequilibrium ionization region was applied to the specific configuration of the plasma thruster, a $\mu$-PPT. It was found that during the thruster operation, both ionization regimes of LTE and nonequilibrium ionization take place.

\section{ACKNOWLEDGMENT}

The first two authors gratefully acknowledge the financial support of the Air Force Office of Scientific Research through Grant No. F49620-02-1-0084.

\section{APPENDIX: ON THE ABLATION MODE}

Ablation modes were considered in Refs. 11, 14, 16, and 17. It was shown that dependent on the density of the background plasma there are two solutions with the same ablation rate. One solution corresponds to the case of ablation into a dense plasma when backflux is very strong. This condition is realized for instance in an electrothermal PPT (Ref. 23), an ablation controlled capillary discharge (Ref. 11), and in the cathode spot of the vacuum arc (Refs. 1 and 15). On the 
other hand, in the case of small background plasma density, the ablation mode is close to that realized in the vacuum case. This mode is realized for instance in an electromagnetic PPT and laser ablation (Refs. 14 and 31).

The main structure that is studied in this respect is the Knudsen layer, which is the layer attached to the surface in which the distribution function of the emitted particles is transformed from a half-Maxwellian into a drifted Maxwellian at the Knudsen layer edge. ${ }^{14}$ This transformation can be viewed as jump conditions in the hydrodynamic description. ${ }^{16}$ The relations between parameters at the Knudsen layer edge are as described by the following set of equations:

$$
\begin{aligned}
\frac{n_{o}}{2\left(\pi d_{o}\right)^{0.5}}= & n_{1} V_{1}+\beta \frac{n_{1}}{2\left(\pi d_{1}\right)^{0.5}}\left\{\exp \left(-\alpha^{2}\right)\right. \\
& \left.-\alpha \pi^{0.5} \operatorname{erfc}(\alpha)\right\},
\end{aligned}
$$

$$
\begin{aligned}
\frac{n_{o}}{4 d_{o}}= & \frac{n_{1}}{2 d_{1}}\left\{\left(1+2 \alpha^{2}\right)-\beta\left[\left(0.5+\alpha^{2}\right) \operatorname{erfc}(\alpha)\right.\right. \\
& \left.\left.-\alpha \exp \left(-\alpha^{2}\right) / \pi^{0.5}\right]\right\},
\end{aligned}
$$

$$
\begin{aligned}
\frac{n_{o}}{\left(\pi d_{o}\right)^{1.5}}= & \frac{n_{1}}{\left(d_{1}\right)^{1.5}} \pi^{-1}\left[\alpha\left(\alpha^{2}+2.5\right)-\beta / 2\{(2.5\right. \\
& \left.\left.\left.+\alpha^{2}\right) \alpha \operatorname{erfc}(\alpha)-\left(2+\alpha^{2}\right) \exp \left(-\alpha^{2}\right) / \pi^{0.5}\right\}\right]
\end{aligned}
$$

where $\quad d_{o}=m / 2 k T_{o}, \quad d_{1}=m / 2 k T_{1}, \quad \alpha=U_{1} /\left(2 k T_{1} / m\right)^{0.5}$; $\operatorname{erfc}(\alpha)=1-\operatorname{erf}(\alpha), \operatorname{erf}(\alpha)$ is the error function, $T_{o}$ is the surface temperature, and $n_{o}$ is the equilibrium density. One can see that in this system of equations, the velocity at the edge of the Knudsen layer remains a free parameter.

Mainly, the ablation mode is determined by the mechanisms of plasma acceleration in the immediate vicinity of the ablated surface. In order to study this effect, the model of the hydrodynamic layer should supplement the Knudsen layer analyses. Using particle and momentum conservation the velocity at the edge of the Knudsen layer can be calculated. This approach was undertaken in Ref. 11 where plasma acceleration in the hydrodynamic layer without magnetic force effects was investigated. To illustrate the effect of the background plasma parameters on the ablation rate, the contours of the ablation rate $(\Gamma)$ in the plasma density-surface temperature plane with electron temperature as a parameter can be calculated. These calculations are shown in Fig. 9. Comparing ablation rates corresponding to several electron temperatures $\left(T_{2}\right)$, one can see that in the case of larger electron temperature the ablation rate decreases. Another interesting effect that is shown in Fig. 9 is that in the low density regime, electron temperature does not affect the ablation rate. On the other hand in the high density regime there is a very strong effect of $T_{2}$ on the ablation rate. This happens because the total ablation rate is the resulting flux between the primary flux and the backflux. In the case of higher $T_{2}$, the backflux increases due to the higher electron temperature in the plasma bulk leading to a decrease in the ablation rate.

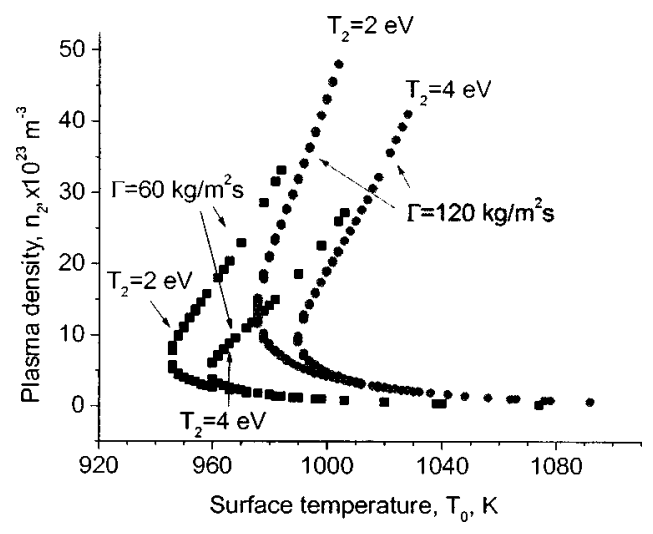

FIG. 9. Ablation rate contours with electron temperature as a parameter.

It seems to be appropriate to mention additional effect related to the ablation mode. In pulsed plasma accelerators as well as in most ablation controlled capillary discharges the Teflon or other compound materials are used. Therefore one can consider an additional effect associated with the polyatomic structure of the vaporized molecular such as an internal degree of freedom. ${ }^{16}$ Change of the internal degree of freedom should affect the energy conservation equation [Eq. (A3)] in the way shown previously. ${ }^{33}$ However, our estimations show that the main portion of the propellant ablates in the monoatomic form under typical conditions in pulsed plasma thrusters. In part, it is due to significant fraction of the atoms that returned back during the quasisteady state operation. $^{12,31}$ In addition it was estimated that this is relatively small effect which can be neglected in comparison with effects of the plasma acceleration, background plasma density, and the electron temperature.

${ }^{1}$ I. I. Beilis, IEEE Trans. Plasma Sci. 29, 657 (2001).

${ }^{2}$ M. Keidar, I. D. Boyd, and I. I. Beilis, IEEE Trans. Plasma Sci. 27, 376 (2001).

${ }^{3}$ L. Muller, J. Phys. D 26, 1253 (1993).

${ }^{4}$ E. Domejean, P. Chevrier, C. Fievet, and P. Petit, J. Phys. D 30, 2132 (1997).

${ }^{5}$ S. V. Kukhlevsky, J. Kaiser, O. Samek, M. Liska, and J. Erostyak, J. Phys. D 33, 1090 (2000).

${ }^{6}$ D. Hong et al., Rev. Sci. Instrum. 71, 15 (2000).

${ }^{7}$ I. I. Beilis, Sov. Phys. Tech. Phys. 19, 288 (1974).

${ }^{8}$ X. Zhou and J. Heberlein, Plasma Sources Sci. Technol. 3, 564 (1994).

${ }^{9}$ B. Rethfeld, J. Wendelstorf, T. Klein, and G. Simon, J. Phys. D 29, 121 (1996).

${ }^{10}$ R. L. Burton and P. Turchi, J. Propul. Power 14, 716 (1998)

${ }^{11}$ M. Keidar, I. D. Boyd, and I. I. Beilis, J. Phys. D 34, 1675 (2001).

${ }^{12}$ M. Keidar, I. D. Boyd, F. S. Gulczinski, III, E. Antonsen, and G. G. Spanjers, J. Propul. Power (in press).

${ }^{13}$ G. G. Spanjers et al., 28th AIAA Joint Propulsion Conference, Indianapolis, IN, USA, July 2002 (American Institute of Aeronautics and Astronautics, Washington DC, 2002), Paper AIAA-2002-3974.

${ }^{14}$ S. I. Anisimov, Sov. Phys. JETP 27, 182 (1968).

${ }^{15}$ I. I. Beilis, Sov. Phys. Dokl. 27, 150 (1982).

${ }^{16}$ C. J. Knight, AIAA J. 17, 519 (1979).

${ }^{17}$ M. Keidar, J. Fan, I. D. Boyd, and I. I. Beilis, J. Appl. Phys. 89, 3095 (2001).

${ }^{18}$ I. Mikellides, Ph.D. thesis, Ohio State University, Columbus, 2000.

${ }^{19}$ P. J. Turchi, I. G. Mikellides, P. G. Mikellides, and H. Kamhawi, in $\mathrm{Mi}$ cropropulsion for Small Spacecraft, Progress in Astronautics and Aeronautics, edited by Michael M. Micci and Andrew D. Ketsdever, Vol. 187, (AIAA, 2000).

${ }^{20} \mathrm{H}$. Alfvén, On the Origin of the Solar System (Oxford University Press, Oxford, 1964).

${ }^{21}$ R. L. Burton et al., in Micropropulsion for Small Spacecraft Progress in 
Astronautics and Aeronautics Vol. 187, edited by Michael M. Micci and Andrew D. Ketsdever (AIAA, 2000).

${ }^{22}$ L. Danielson, Phys. Fluids 13, 2288 (1970).

${ }^{23}$ M. Keidar, I. D. Boyd, and I. I. Beilis, J. Propul. Power 19, 424 (2003). ${ }^{24}$ D. E. Potter, Phys. Fluids 14, 1911 (1971).

${ }^{25}$ A. Fruchtman, N. J. Fisch, and Y. Raitses, Phys. Plasmas 8, 1048 (2001).

${ }^{26}$ E. Ahedo, P. Martinez-Cerezo, and M. Martinez-Sanchez, Phys. Plasmas 8, 3058 (2001).

${ }^{27}$ A. I. Zemskov, V. V. Prut, and V. A. Khrabrov, Sov. Phys. Tech. Phys. 17, 285 (1972).
${ }^{28}$ G. I. Kozlov, V. A. Kuznetsov, and V. A. Masyukov, Sov. Phys. JETP 39, 463 (1974).

${ }^{29}$ Y. P. Raizer, Gas Discharge Physics (Nauka, Moscow, 1987) (in Russian).

${ }^{30}$ A. S. Kingsep, Y. V. Mokhov, and K. V. Chukbar, Sov. J. Plasma Phys. 10, 495 (1984).

${ }^{31}$ M. Keidar, I. D. Boyd, E. Antonsen, and G. G. Spanjers, J. Propul. Power (in press).

${ }^{32}$ IAEA Aladin database (http://www-amdis.iaea.org/aladdin.html).

${ }^{33}$ R. L. Baker, AIAA J. 29, 471 (1991). 